ARGONNE NATIONAL LABORATORY

9700 South Cass Avenue

Argonne, Illinois 60439

\title{
CLADDING FAILURE BY LOCAL PLASTIC INSTABILITY
}

by

J. M. Kramer and L. W. Deitrich

Reactor Analysis and Safety Division

December 1977

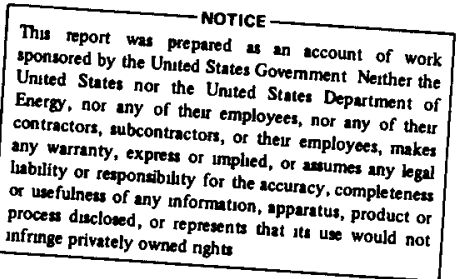

declosed, or represention, apparatus, product or

infruge privately owned nghts 


\section{DISCLAIMER}

This report was prepared as an account of work sponsored by an agency of the United States Government. Neither the United States Government nor any agency Thereof, nor any of their employees, makes any warranty, express or implied, or assumes any legal liability or responsibility for the accuracy, completeness, or usefulness of any information, apparatus, product, or process disclosed, or represents that its use would not infringe privately owned rights. Reference herein to any specific commercial product, process, or service by trade name, trademark, manufacturer, or otherwise does not necessarily constitute or imply its endorsement, recommendation, or favoring by the United States Government or any agency thereof. The views and opinions of authors expressed herein do not necessarily state or reflect those of the United States Government or any agency thereof. 


\section{DISCLAIMER}

Portions of this document may be illegible in electronic image products. Images are produced from the best available original document. 

TABLE OF CONTENTS

$\underline{\text { Page }}$

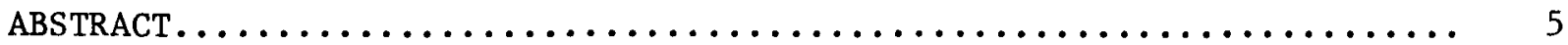

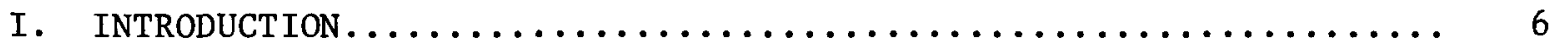

II. BULGE-DEFORMATION KINEMATICS $\ldots \ldots \ldots \ldots \ldots \ldots \ldots \ldots \ldots \ldots \ldots \ldots$

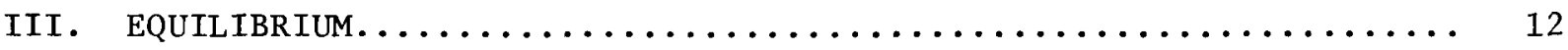

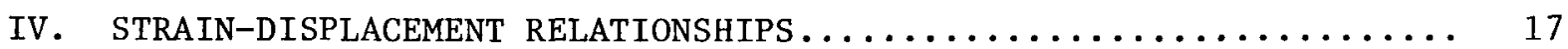

V. CONSTITUTIVE EQUATIONS - AN EXAMPLE................... 19

VI. RESULTS AND DISCUSSION.......................... 24

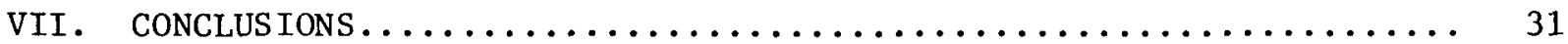

ACKNOWLEDGMENTS ............................... 33

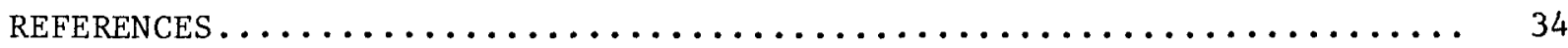




\section{LIST OF FIGURES}

$\underline{\text { Page }}$

Figure 1. Cladding Geometry: (A) Undeformed; (B) Deformed . . . 7

Figure 2. Differential Element of Deformed Middle Surface . . . 9

Figure 3. Biaxial Stress-rupture Strength of 10-15\%

Cold-worked Stainless Stee1, Data (symbols)

from D. F. Atkins [4]. $(1 \mathrm{~h}=3600 \mathrm{~s}) . . . . . .25$

Figure 4. Dimensionless Radial (a), Axial $(\hat{\lambda})$, and Bulge $(\hat{\varepsilon})$

Displacements of a Closed Tube for an Initial 0.0055-mmamplitude Bulge of Length $10.0 \mathrm{~mm}$ and No Temperature Percurbation. (1 hr $=3600 \mathrm{~s})$. . . . . . . . 27

Figure 5. Same as Fig. 4, Except with an Initial Bulge Length of $5.0 \mathrm{~mm}$. (1 hr $=3600 \mathrm{~s})$.......... 28

Figure 6. Same as Fig. 4, Except for an Open Tube with No Axial Force. $(1 \mathrm{hr}=3600 \mathrm{~s}) . . . . . . . . .28$

Figure 7. Same as Fig. 4, Except with $10^{\circ} \mathrm{C}$ Temperature Perturbation. (1 hr $=3600 \mathrm{~s}) . .$. . . . . . 29

LIST OF TABLES

Table I Stainless Steel Properties Used in Equation 5-5. . . . . . 24 
CLADDING FAILURE BY LOCAL PLASTIC INSTABILITY

by

J. M. Kramer and L. W. Deitrich

ABSTRACT

Cladding failure is one of the major considerations in analysis of fuel-pin behavior during hypothetical accident transients since time, location, and nature of failure govern the early postfailure material motion and reactivity feedback. Out-of-pile thermal transient tests of both irradiated and unirradiated fast-reactor cladding show that local plastic instability, or bulging, often precedes rupture and that the extent of local instability limits the initial rip length.

To investigate the details of bulge formation and growth, a perturbation analysis of the equations governing large deformation of a cylindrical shell has been developed, resulting in a set of linear differential equations for the bulge geometry. These equations have been solved along with appropriate constitutive equations and various constraints on the ends of the cladding.

Sources for bulge formation that have been considered include initial geometric imperfections and thermal perturbations due to either eccentric fuel pellets or nonsymmetric cooling. Of these, only the first is relevant to out-of-pile burst tests. Here it has been found that the most likely imperfection that will grow unstably to failure leads to a bulge around half the circumference with an axial length 1.1 times the deformed diameter. This is in general agreement with burst-test results. For the case of in-reactor fuel pins, it has been found that thermal perturbations can significantly affect local instability, particularly if the deformation process is thermally activated with a high activation energy. 


\section{INTRODUCTION}

The response of cladding to mechanical loads is one of the major considerations in the analysis of fuel behavior during hypothetical accident transients in fast breeder reactors. Failure of cladding under these loads is important in both overpower and undercooling accident sequences. In the overpower accident, mechanical loads lead to cladding failure allowing the initial release of fuel and fission gas from the pin. The release of fuel and its subsequent motion can lead to early neutronic shutdown of the reactor in the location of the failure is favorable - such that fuel motion is away from the core midplane or to an energetic excursion if the location of failure is unfavorable. On the other hand, cladding failure in the high-power subassemblies in an undercooling situation usually results from cladding melting. Under some conditions, however, reactivity feedbacks due to sodium voiding and cladding relocation in undercooled subassemblies can lead to severe power excursions in other subassemblies not yet voided. The behavior of fuel in these subassemblies is expected to be similar to that in the overpower accident, although important differences may arise due to differences in temperature distribution.

Loading of the cladding in an overpower accident may result from differenLial thermal expansion of fuel and cladding, from transient fuel swelling, from molten-fuel expansion, or from pressure of fission gas initially in the porosity or released from grains during the transient. The present analysis considers the response of cladding to those loads that act hydrostatically. This type of loading is thought to be most representative of conditions prior to cladding failure during transient overpower conditions [1]. The analysis is also directly applicable to recent experiments [2] on both fresh and irradiated stainless steel FTR cladding in which gas pressure loading was used.

The transient burst experiments [2] have shown that high-temperature failure of cladding is often by local plastic instability or bulging. This deformation is illustrated in Fig. 1B. A small section of the cladding, which may be the site of either a geometric imperfection or a thermal hot spot, grows more rapidly than the remainder of the cladding. The strains in this localized bulge are larger than the average strains. If temperatures are high and strain rates low, grain-boundary separation may lead to a pin-hole failure at this site. On the other hand, if a pin-hole failure does not occur, or if the 
loading is maintained, the bulge may eventually begin to grow unstably, leading to rapid rupture by ripping along the length of the bulge. In either case, the failure is governed by the bulge behavior. It is the analysis of this localized behavior that is the subject of this paper.

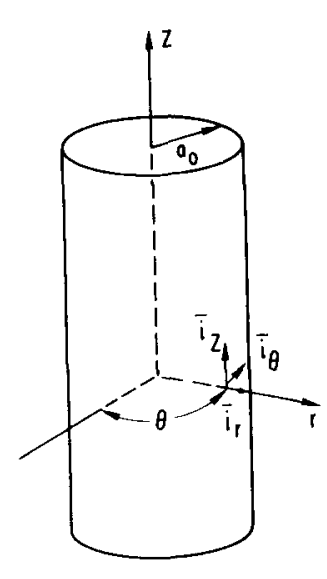

(a)

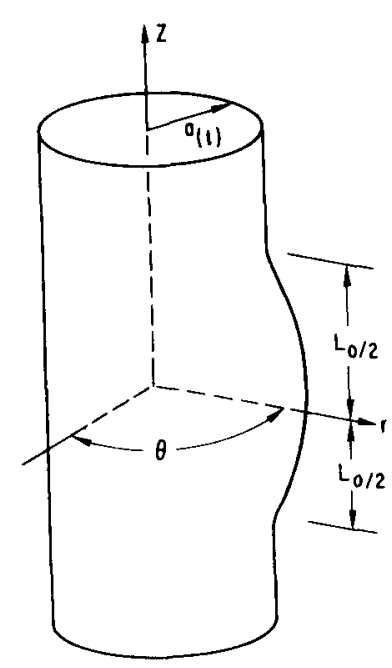

(b)

Fig. 1 Cladding geometry: (A) Undeformed; (B) Deformed. 


\section{BULGE-DEFORMATION KINEMATICS}

We model the cladding as a thin cylindrical shell of radius $a_{0}$ and thickness $h_{0}$ subjected to an internal pressure $P$. The middle surface of the shell in the undeformed state is described by $r=a_{0}$, where $(r, \theta, z)$ is the cylindrical coordinate system shown in Fig. $1 \mathrm{~A}$ along with the associated unit vectors $\left(\bar{i}_{r}, \bar{i}_{\theta}, \bar{i}_{z}\right)$.

During deformation, at some time $t$, a material particle which occupied coordinates $\theta_{0}, z_{0}$ on the undeformed middle surface will now occupy the spatial position given by

$$
\begin{aligned}
& r=\mathrm{f}_{1}\left(\theta_{0}, \mathrm{z}_{0}, \mathrm{t}\right) ; \\
& \theta=\mathrm{f}_{2}\left(\theta_{0}, \mathrm{z}_{0}, \mathrm{t}\right) ; \\
& z=\mathrm{f}_{3}\left(\theta_{0}, z_{0}, t\right)
\end{aligned}
$$

as shown in Fig. 1B. With this notation, $\theta_{0}$ and $z_{0}$ serve both as labels for material particles and as parameters to characterize the deformed middle surface. Since for all times $t$ fixed values of these parameters locate the spatial position of the same material particle, $\theta_{0}$ and $z_{0}$ are often referred to as convected coordinates. Once the forms of $f_{1}, f_{2}$, and $f_{3}$ in Eqs. 2-1 are explicitly known, all of the properties of the deformation of the shell, such as stretching, thickness change, and curvature change, can be determined.

In order to proceed further, we simplify the kinematics by assuming that the deformation consists of a local perturbation, or bulge, super-imposed on an axisymmetric deformation which transforms the undeformed cylinder of radius $a_{0}$ into another cylinder of radius $a(t)$. It is further assumed that the overall deformation is plane in the axial direction and that the radial displacement is much larger than the tangential displacement. Equations 2-1 can then be reduced to

$$
\begin{aligned}
& r=a(t)+\varepsilon\left(\theta_{0}, z_{0}, t\right) ; \\
& \theta=\theta_{0} ;
\end{aligned}
$$




$$
z=\lambda(t) z_{0}
$$

with the position vector of the deformed middle surface given by

$$
\bar{r}=(a+\varepsilon) \bar{i}_{r}+\lambda z_{o} \bar{i}_{z}
$$

where $\varepsilon$ is the perturbation to the cylindrical geometry. Although the simplified kinematics described by Eqs. 2-2 is plausible in light of the localized nature of the deformation shown in Fig. 1B, its validity, of course, can only be tested a posteriori from experimental evidence.

We consider now the geometry of a differential element of the deformed middle surface as shown in Fig. 2. The covariant base vectors $\overline{\mathrm{e}}_{1}$ and $\bar{e}_{2}$, which 1 ie on the surface along the $\theta_{0}$ and $z_{0}$ surface coordinates, are given from Eq. $2-3$ by

$$
\begin{aligned}
& \overline{\mathbf{e}}_{1}=\frac{\partial \bar{r}}{\partial \theta_{0}}=\frac{\partial \varepsilon}{\partial \theta_{o}} \overline{\mathbf{i}}_{r}+(a+\varepsilon) \bar{i}_{\theta}, \\
& \bar{e}_{2}=\frac{\partial \bar{r}}{\partial z}=\frac{\partial \varepsilon}{\partial z} \bar{i}_{o}+\lambda \bar{i}_{z} .
\end{aligned}
$$

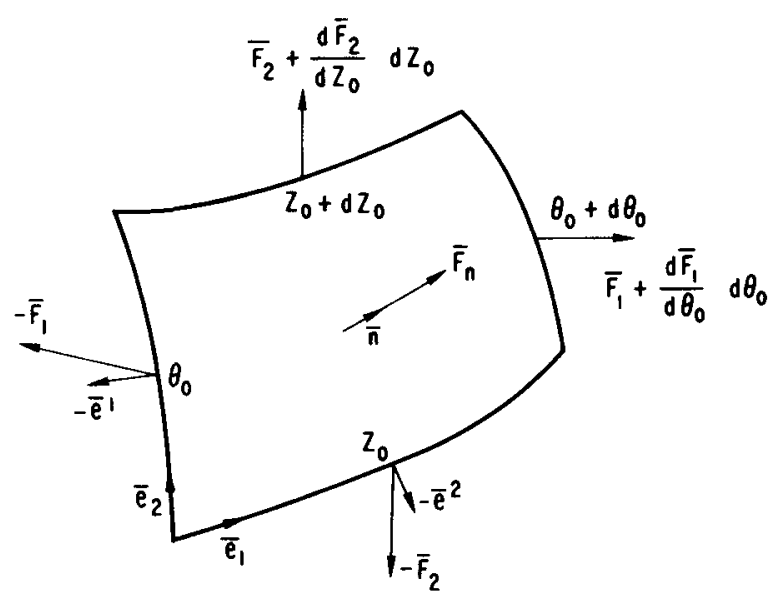

Fig. 2 Differential Element of Deformed Middle Surface. 
The differential vector $\overline{d \bar{r}}$ connecting two neighboring points on the deformed surface is defined by

$\overline{\mathrm{d}} \overline{\mathrm{r}}=\frac{\partial \overline{\mathrm{r}}}{\partial \theta_{0}} \mathrm{~d} \theta_{0}+\frac{\partial \overline{\mathbf{r}}}{\partial \mathrm{z}_{\mathrm{o}}} \mathrm{dz} \mathrm{o}_{0}$

and the separation ds of these two points is given by

$$
\mathrm{ds} s^{2}=\overline{\mathrm{dr}} \cdot \mathrm{d} \overline{\mathrm{r}}=E \mathrm{Ed} \theta_{\mathrm{o}}^{2}+2 \mathrm{Fd} \theta_{\mathrm{o}} \mathrm{d} z_{\mathrm{o}}+\mathrm{Gd} z_{\mathrm{o}}^{2}
$$

where $\mathrm{E}, \mathrm{F}$, and $\mathrm{G}$ are the first fundamental magnitudes of the surface.*

Substitution of Eqs. $2-4$ and 2.5 into Eq. 2-6 shows that

$$
\begin{aligned}
& E=\left(\frac{\partial \varepsilon}{\partial \theta_{0}}\right)^{2}+(a+\varepsilon)^{2}, \\
& F=\frac{\partial \varepsilon}{\partial z_{o}} \frac{\partial \varepsilon}{\partial \theta_{o}}, \\
& G=\left(\frac{\partial \varepsilon}{\partial z_{0}}\right)^{2}+\lambda^{2} .
\end{aligned}
$$

The surface area $\mathrm{dA}$ and the unit normal $\overline{\mathrm{n}}$ of the differential element shown in Fig. 2 can be determined from

$$
\mathrm{dA} \overline{\mathrm{n}}=\overline{\mathrm{e}}_{1} \times \overline{\mathrm{e}}_{2} \mathrm{~d} \theta_{\mathrm{o}} \mathrm{dz} \mathrm{o}_{\mathrm{o}}=\operatorname{Hd} \theta_{\mathrm{o}} \mathrm{d} \mathrm{z}_{\mathrm{o}} \overline{\mathrm{n}}
$$

where $\mathrm{H}$ is found by substituting Eqs. 2-4 into Eq. 2-8, or equivalently, from Eqs. $2-7$ by

$$
H=\sqrt{E G-F^{2}} \text {. }
$$

The unit normal $\overline{\mathrm{n}}$ is then given by

$$
\overline{\mathrm{n}}=\frac{\mathrm{e}_{1} \times \mathrm{e}_{2}}{\mathrm{H}}
$$

*For a more complete discussion of differential geometry, see for instance [3]. 
The curvature of the deformed middle surface is described by the second fundamental magnitudes $L, M$, and $N$; whose definition is

$$
\begin{aligned}
& L=-\frac{\partial \overline{\mathrm{e}}_{1}}{\partial \theta_{0}} \cdot \overline{\mathrm{n}}, \\
& \mathrm{M}=-\frac{\partial \overline{\mathrm{e}}_{1}}{\partial \mathrm{z}_{\mathrm{o}}} \cdot \overline{\mathrm{n}}=-\frac{\partial \overline{\mathrm{e}}_{2}}{\partial \theta_{0}} \cdot \overline{\mathrm{n}}, \\
& \mathrm{N}=-\frac{\partial \overline{\mathrm{e}}_{2}}{\partial \bar{z}_{\mathrm{o}}} \cdot \overline{\mathrm{n}} .
\end{aligned}
$$

Substituting Eqs. 2-4 into these definitions gives

$$
\begin{aligned}
& L=\left[-(a+\varepsilon)\left(\frac{\partial^{2} \varepsilon}{\partial \theta_{0}^{2}}-a-\varepsilon\right)+2 \frac{\partial \varepsilon^{2}}{\partial \theta_{o}}\right] \frac{\lambda}{H} \\
& M=\left[-(a+\varepsilon) \frac{\partial^{2} \varepsilon}{\partial \theta_{o} \partial z_{o}}+\frac{\partial \varepsilon}{\partial \theta_{o}} \frac{\partial \varepsilon}{\partial z_{o}}\right] \frac{\lambda}{H} \\
& N=-(a+\varepsilon) \frac{\partial^{2} \varepsilon}{\partial z_{o}^{2}} \frac{\lambda}{H} .
\end{aligned}
$$


III. EQUILIBRIUM

Mechanical equilibrium of the cladding in the absence of bulging $(\varepsilon=0)$ is maintained by membrane forces. The only bending stresses come from selfequilibrating thermal and swelling stresses, and these tend to relax due to thermal and fission-enhanced creep. We assume here that the situation is not significantly different in the perturbed geometry in that the pressure loading is still carried primarily by membrane forces and it is these forces that determine whether the bulge will grow unstably and lead to local failure. Since bulge growth occurs at high temperatures where significant plastic flow is possible, this assumption appears reasonable.

The only relevant equilibrium equation then comes from the summation of forces normal to the middle surface.

The membrane stresses $\overline{\bar{\sigma}}$ acting on a differential shell element of thickness $h$ whose middle surface is shown in Fig. 2 can be written in dyadic notation* as

$$
\overline{\bar{\sigma}}=\sigma^{i j} \bar{e}_{i} \bar{e}_{j} \quad i, j=1,2
$$

with the membrane force resultants (force/unit length) $\overline{\mathrm{f}}_{1}, \overline{\mathrm{f}}_{2}$ acting on the faces of the differential element given by

$$
\begin{aligned}
& \overline{\mathrm{f}}_{1}=h \overline{\bar{\sigma}} \cdot \frac{\overline{\mathrm{e}^{1}}}{\left|\overline{\mathrm{e}}^{1}\right|} \\
& \overline{\mathrm{f}}_{2}=\mathrm{h \overline { \sigma }} \cdot \frac{\overline{\mathrm{e}^{2}}}{|\overline{\mathrm{e}}|}
\end{aligned}
$$

Here $\overline{\mathrm{e}}^{1}$ and $\overline{\mathrm{e}}^{2}$ are the contravariant base vectors defined as being orthonormal to $\bar{e}_{2}$ and $\bar{e}_{1}$ as shown in Fig. 2. Consequently, the terms to the right of the inner products in Eqs. 3-2 are nothing more than the unit normals to the coordinate faces. Furthermore, the lengths of the coordinate faces are $\sqrt{\mathrm{E}} \mathrm{d} \theta_{0}$ and $\sqrt{\mathrm{F}} \mathrm{d} \mathrm{z}_{\mathrm{o}}$, as can easily be seen from $\mathrm{Eq}$. 2-6 by successively setting $\mathrm{d} z_{0}$ and $\mathrm{d} \theta_{\mathrm{o}}$ equal to zero. The total forces $\overline{\mathrm{F}}_{1}$ and $\overline{\mathrm{F}}_{2}$ acting on coordinate

\footnotetext{
"Summation notation will be used in tensor equations. Indices 1 and 2 refer to $\theta_{0}$ and $z_{0}$, respectively.
} 
faces shown in Fig. 2 are then given by Eqs. 3-1 and 3-2 as

$$
\begin{aligned}
& \overline{\mathrm{F}}_{1}=\operatorname{Hh}\left[\sigma^{1} \overline{\mathrm{e}}_{1}+\sigma^{21} \overline{\mathrm{e}}_{2}\right] \mathrm{d} \mathrm{z}_{\mathrm{o}} ; \\
& \overline{\mathrm{F}}_{2}=\operatorname{Hh}\left[\sigma^{12} \overline{\mathrm{e}}_{1}+\sigma^{22} \overline{\mathrm{e}}_{2}\right] \mathrm{d} \theta_{\mathrm{o}} ;
\end{aligned}
$$

where we have made use of the fact that from the definition of $\bar{e}^{l}$ and $\bar{e}^{2}$,

$$
|\overline{\mathrm{e}}|=\sqrt{\mathrm{G}} / \mathrm{H}
$$

and

$$
\left|\overline{\mathrm{e}}^{2}\right|=\sqrt{\mathrm{E}} / \mathrm{H}
$$

Besides the forces $\bar{F}_{1}$ and $\vec{F}_{2}$, the only other net force acting on the she11 element comes from the pressure P. From Eq. 2-8, this force $\bar{F}_{n}$ is simply

$$
\bar{F}_{n}=P H d \theta_{0} d z_{0} \bar{n}
$$

It is now possible to sum all of the vector forces shown in Fig. 2 to satisfy equilbrium. In general, this sumation will require the derivatives of Eq. 3-3a and Eq. 3-3b with respect to $\theta_{0}$ and $z_{0}$. However, the only equation that is of interest is the normal component of the vector sum of forces, which can be found by taking the inner product of the sum with the unit normal. Since $\bar{n}$ is orthogonal to $\overline{\mathbf{e}}_{1}$ and $\overline{\mathbf{e}}_{2}$, the only contributions to this equation will come from Eq. $3-5$ and from the inner product of $\bar{n}$ with the derivatives of $\overline{\mathrm{e}}_{1}$ and $\overline{\mathrm{e}}_{2}$ in Eqs. 3-3a and 3-3b. These inner products are just the definition of $\mathrm{L}, \mathrm{M}$, and $\mathrm{N}$ in Eqs. 2-11. Therefore the summation of forces normal to the she11 gives

$$
L \sigma^{11}+2 M \sigma^{12}+N \sigma^{22}=\frac{P}{h}
$$

It is useful to note here that when $\varepsilon$ is zero, substitution of Eqs. 2-12, 2-9, and 2-7 into Eq. 3-6 gives

$$
\sigma_{a}^{11}=\frac{P}{a h_{a}}
$$


where the subscript "a" will be used to denote conditions when there is no perturbation. This result can be expressed in more familiar form by introducing the physical components of the stress tensor $\sigma^{(i)}(j)$ referred to the unit vectors $\bar{e}_{i} /\left|\bar{e}_{i}\right|$, since $i t$ is these components that have the physical units of stress (force/length ${ }^{2}$ ). The right physical components $\sigma_{(j)}^{(i)}$ are defined in dyadic notation by

$$
\overline{\bar{\sigma}}=\sigma_{\text {(j) }}^{(i)} \bar{e}_{i} \bar{e}^{-j} \frac{\left|\bar{e}_{j}\right|}{\left|\bar{e}_{i}\right|} ; i, j=1,2 .
$$

By equating the right-hand sides of Eqs. 3-1 and 3-8, and making use of the orthonormality of the two sets of base vectors, the relationships between $\sigma^{i j}$ and $\sigma_{(j)}^{(i)}$ can be extracted:

$$
\begin{aligned}
& \sigma_{(1)}^{(1)}=\mathrm{E} \sigma^{11}+\mathrm{F} \sigma^{12}, \\
& \sigma_{(2)}^{(1)^{2}}=\sqrt{\frac{E}{G}}\left(F \sigma^{11}+G \sigma^{12}\right) ; \\
& \sigma_{(2)}^{(2)}=F \sigma^{21}+G \sigma^{22}
\end{aligned}
$$

Equations 3-7, 3-9a, and 2-7 then give the familiar result for the hoop stress in a cylindrical shell:

$$
\sigma_{a}^{(1)}(1)=\frac{\mathrm{Pa}_{\mathrm{a}}}{\mathrm{h}_{\mathrm{a}}}
$$

It is also noted here that from elementry considerations of a cylindrical shell under internal pressure

$$
\sigma_{\mathrm{a}}^{(1)}(2)=0
$$

and

$$
\sigma_{a}^{(2)}(2)=\sigma_{z a}
$$


If the ends of the cylinder are capped, $\sigma_{z a}$ is just half the hoop stress given by Eq. 3-10.

Consider now the equilibrium equation 3-6 for the perturbed geometry. The stress $\overline{\bar{\sigma}}$ will differ from the unperturbed stress $\overline{\bar{\sigma}}_{a}$ by an amount $\overline{\bar{\sigma}}_{\varepsilon}$ which must tend to zero as $\varepsilon$ tends to zero, or

$$
\overline{\bar{\sigma}}=\overline{\bar{\sigma}}_{\mathrm{a}}+\overline{\bar{\sigma}}_{\varepsilon}
$$

Also

$$
\sigma^{i j}=\sigma_{a}^{i j}+\sigma_{\varepsilon}^{i j}
$$

and

$$
\sigma_{(j)}^{(i)}=\sigma_{a}^{(i)}(j)+\sigma_{\varepsilon}^{(i)}(j)
$$

Substituting these stress perturbations and $L, M$, and $N$ from Eqs. 2-12 into Eq. 3-6, and neglecting terms of the order $|\varepsilon|^{2}$, gives

$$
\sigma_{\varepsilon}^{(i)_{(j)}}=\frac{P \varepsilon}{h_{a}}-\frac{P a h}{h_{a}^{2}}+\frac{P}{h_{a}} \frac{\partial^{2} \varepsilon}{\partial \theta_{o}^{2}}+\frac{\sigma_{z a} a^{a}}{\lambda^{2}} \frac{\partial^{2} \varepsilon}{\partial z_{o}^{2}}
$$

where Eqs. 3-9 have been used to relate the stress components, and the zeroth order approximations have been substituted from Eqs. 3-10, 3-11, and 3-12. It is worth noting that only the hoop-stress perturbation gives a first-order contribution to the equilibrium of the shell. The first two terms of the right-hand side of Eq. 3-16 represent a hoop-stress perturbation due to the change in radius and wall thickness, while the second two terms are the contributions due to the curvature changes.

The remaining equations necessary to complete the formulation came from the constitutive equations, which relate the stress perturbation ${ }_{\varepsilon}(1)(1)$ to the perturbed deformation history $\left(\varepsilon\right.$ and $\left.h_{\varepsilon}\right)$. However, if the deformation is predominantly plastic, these equations can be simplified by assuming that the material is incompressible. The volume $\mathrm{dV}$ of the differential element shown in Fig. 2 is given by Eq. $2-8$ as

$$
\mathrm{dV}=\mathrm{hdA}=\mathrm{hHd} \mathrm{z}_{\mathrm{o}} \mathrm{d} \theta_{\mathrm{o}}
$$


In the undeformed state, the volume $\mathrm{dV}_{\mathrm{o}}$ of this same material element is

$$
d V_{0}=h_{0} a_{0} d z_{0} d \theta_{0}
$$

Substituting

$$
\mathrm{h}=\mathrm{h}_{\mathrm{a}}+\mathrm{h}_{\varepsilon}
$$

along with Eqs. 2-9 and 2-7, and neglecting terms of the order $|\varepsilon|^{2}$, gives

$$
\begin{aligned}
& h_{a}=\frac{h_{o} a_{0}}{a \lambda} ; \\
& h_{\varepsilon}=-\varepsilon \frac{h_{o} a_{0}}{a^{2} \lambda} .
\end{aligned}
$$

Substituting these equations into Eq. 3-16 yields

$$
\sigma_{\varepsilon}^{(1)}(1)=\frac{2 \operatorname{Pa} \lambda \varepsilon}{h_{0} a_{0}}+\frac{P a \lambda}{h_{o} a_{0}} \frac{\partial^{2} \varepsilon}{\partial \theta_{o}^{2}}+\sigma_{z a} \frac{a}{\lambda^{2}} \frac{\partial^{2} \varepsilon}{\partial z_{o}^{2}}
$$

for an incompressible material. 


\section{STRAIN-DISPLACEMENT RELATIONSHIPS}

The Lagrangian strain tensor $E_{k \ell}$ serves as a finite strain measure of the relative deformation of neighboring material particles. Considering for the moment only membrane strains, $\mathrm{E}_{\mathrm{k} \ell}$ is defined by

$$
\mathrm{ds}^{2}-\mathrm{ds}_{\mathrm{o}}^{2}=2 \mathrm{E}_{\mathrm{k} \ell} \mathrm{dx}^{\mathrm{k}} \mathrm{dx} \mathrm{x}^{\ell} ; \quad \mathrm{k}, \ell=1,2,
$$

where $d_{s}$ and $d_{s}$ are the separation of neighboring material particles in the deformed and undeformed states, and $\left(X^{1}, X^{2}\right)$ represent the convected coordinates $\left(\theta_{0}, z_{0}\right)$. Substituting Eqs. $2-6$ and 2-7 into Eq. 4-1, and neglecting terms of the order $|\varepsilon|^{2}$ gives

$$
\begin{aligned}
& 2 E_{11}=a^{2}-a_{o}^{2}+2 a \varepsilon \\
& 2 E_{12}=2 E_{21}=0 ; \\
& 2 E_{22}=\lambda^{2}-1
\end{aligned}
$$

Equation 4-2b shows that to the first order of approxiamtion there is no shear distortion of the middle surface. It is therefore possible to introduce the more familiar engineering large-strain measure $\varepsilon_{(i)}^{\text {(i) }}$ - the so-called "true" strain - defined as the natural logarithm of the stretches ds/ds along the coordinate directions. These ratios can also be determined from Eqs. 2-6 and 2-7. Again neglecting terms of the order $|\varepsilon|^{2}$,

$$
\begin{aligned}
& \varepsilon_{(1)}^{(1)}=\varepsilon_{\theta_{0}}=\ln \left(\frac{a}{a_{0}}\right)+\frac{\varepsilon}{a} ; \\
& \varepsilon_{(2)}^{(2)}=\varepsilon_{z_{0}}=\ln \lambda .
\end{aligned}
$$

The third principal strain $\varepsilon^{(3)}$ (3) perpendicular to the middle surface is simp1y

$$
\varepsilon_{(3)}^{(3)}=\ln \left(\frac{h_{a}}{h_{0}}\right)+\frac{h_{\varepsilon}}{h_{a}}
$$


Since most engineering data on the large-strain behavior of materials is presented in terms of "true" strain, this definition of strain will be utilized in subsequent analysis.

It has been assumed in Eqs. 4-3 that the stress-free state is a perfect cylinder of radius $a_{0}$ and that this state serves as a reference for zero strain. In fact, however, there will always exist initial imperfections in the geometry. Within the framework of the present analysis, these imperfections can be modeled as initial perturbations $\varepsilon_{0}\left(\theta_{0}, z_{0}\right)$ and $h_{\varepsilon_{0}}\left(\theta_{0}, z_{0}\right)$. The strains then become

$\varepsilon_{(1)}^{(1)}=\ln \left(\frac{\mathrm{a}}{\mathrm{a}_{\mathrm{o}}}\right)+\frac{\varepsilon}{\mathrm{a}}-\frac{\varepsilon_{\mathrm{o}}}{\mathrm{a}_{\mathrm{o}}}$;

$\varepsilon_{(2)}^{(2)}=\ln \lambda ;$

$\varepsilon_{(3)}^{(3)}=\ln \left(\frac{h_{a}}{h_{o}}\right)+\frac{h_{\varepsilon}}{h_{a}}-\frac{h_{\varepsilon o}}{h_{o}}$ 


\section{CONSTITUTIVE EQUATIONS - AN EXAMPLE}

In this section, the perturbation analysis that was developed in preceding sections is applied to cladding whose constitutive equation is assumed to have a particular form, although many of the results will be of a more general nature. The analysis for a different set of constitutive equations is a straightforward exercise. In fact, since the perturbation equations are always linear, the most difficult part of the problem is the determination of the zeroth-order approximation for the unperturbed stresses and strains in a perfect cylindrical shell.

It is assumed here that the cladding material is incompressible and obeys the Prandtl-Reuss flow equations, and that the elastic contributions to the strains are negligible. The differential strain increment $d \varepsilon_{(j)}^{(i)}$ is then

$$
\mathrm{d}^{(i)}(j)=\frac{3}{2} \mathrm{~S}_{(j)}^{(i)} \frac{\mathrm{d} \bar{\varepsilon}}{\bar{\sigma}} ; \quad i, j=1,2,3
$$

where $S^{(i)}(j)$ is the deviatoric component of the stress, defined by

$$
\begin{aligned}
& S_{(j)}^{(i)_{(j)}}=\sigma_{(i)}^{(j)} i, j=1,2,3 ; \\
& \sigma=\frac{1}{3}\left[\sigma_{(1)}^{(1)}+\sigma_{(2)}^{(2)}+\sigma_{(3)}^{(3)}\right],
\end{aligned}
$$

and $\bar{\sigma}$ and $d \bar{\varepsilon}$ are the equivalent stress and equivalent strain increment. In the absence of shear strains, $\bar{\sigma}$ and $\mathrm{d} \bar{\varepsilon}$ are given by

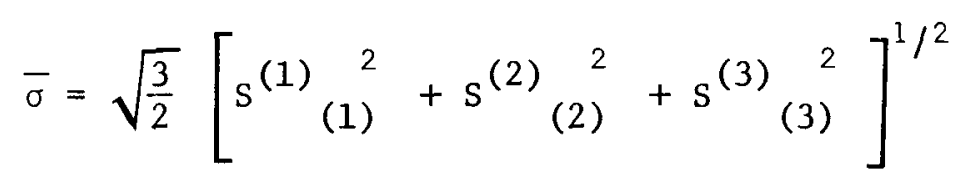

and

$$
\mathrm{d} \bar{\varepsilon}=\sqrt{\frac{2}{3}}\left[\mathrm{~d} \varepsilon_{(1)^{(1)}{ }^{2}+\mathrm{d}^{(2)}{ }_{(2)}^{2}+\mathrm{d}^{(3)}{ }_{(3)}^{2}}\right]^{1 / 2}
$$


It is further assumed that the temperature of the cladding is sufficiently high such that time-dependent plastic strains are dominant and that these strains can be represented by a power-1aw function of the form

$$
\frac{\mathrm{d} \bar{\varepsilon}}{\mathrm{dt}}=\mathrm{C} \bar{\sigma} \mathbf{n}
$$

where $C$ and $n$ are temperature-dependent material constants. Substitution of Eq. 5-5 into Eq. 5-1 gives

$$
\frac{\mathrm{d}^{(i)}(j)}{\mathrm{dt}}=\frac{3}{2} \mathrm{C} \bar{\sigma}^{\mathrm{n}-1_{S}(i)}(j) ; \quad i, j=1,2,3 \text {. }
$$

The constitutive equation 5-6 can be reduced to a zeroth-order of approximation and a first-order perturbation in the same manner that the equations in Sects. 3 and 4 were reduced. The zeroth-order equation is obviously

$$
\frac{\mathrm{d} \varepsilon_{\mathrm{a}}^{(i)}(j)}{\mathrm{dt}}=\frac{3}{2} \overline{C \sigma}_{\mathrm{a}}^{\mathrm{n}-1} \mathrm{~s}_{\mathrm{a}}{ }^{(i)}(j) \quad ; \quad i, j=1,2,3
$$

where again the subscript "a" refers to quantities when $\varepsilon=0$. The relationship between the strain perturbation $\varepsilon_{\varepsilon}^{(i)}(j)$ and the stress perturbations $s_{\varepsilon}^{(i)}(j)$ can easily be formed by substituting into Eq. 5-6 the stress from Eqs. 3-15 and the strains from Eqs. 4-4 and 3-20. Neglecting terms of the order $|\varepsilon|^{2}$, and making use of Eq. 5-7 shows that

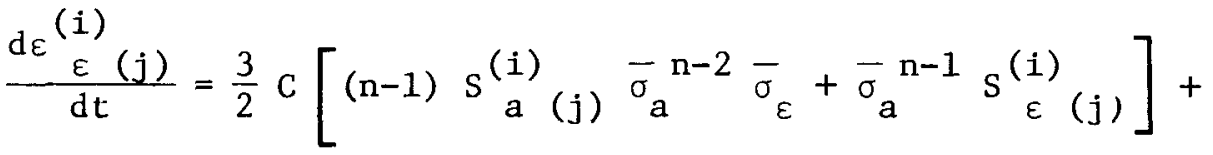

$$
\begin{aligned}
& \frac{3}{2} \mathrm{c}_{\varepsilon} \bar{\sigma}_{\mathrm{a}}^{\mathrm{n}-1} \mathrm{~s}_{\mathrm{a}(\mathrm{j})}^{(\mathrm{i})} \quad ; \quad \mathbf{i , j}=1,2,3
\end{aligned}
$$

where

$$
\bar{\sigma}_{\varepsilon}=\frac{3}{2} \underset{\sigma_{\mathrm{a}}}{\frac{1}{\sigma^{-}}}\left[\begin{array}{c}
\mathrm{S}_{\mathrm{a}}^{(1)} \\
\sigma_{\varepsilon}^{(1)}(1)
\end{array} \mathrm{S}_{\mathrm{a}}^{(2)}{ }_{(2)}^{\sigma_{\varepsilon}^{(2)}(2)}\right] \text {. }
$$


It has also been assumed here that only the material constant $C$ shows a strong temperature dependence. The parameter $C_{\varepsilon}$ then represents the perturbation of $C$ due to small temperature nonuniformities.

In order to be as general as possible, the cladding is assumed to be loaded axially by a force $F_{z}$ and by gas pressure acting on closed ends. The axial stress $\sigma_{z a}$ in Eq. 3-12 is

$$
\sigma_{z a}=\frac{P a}{2 h_{a}}+\frac{F_{z}}{2 \pi a h_{a}},
$$

or, making use of the incompressibility condition 3-20a

$$
\sigma_{z a}=\frac{P a^{2} \lambda}{2 a_{o} h_{o}}+\frac{F_{z} \lambda}{2 \pi a_{o} h_{o}} .
$$

The deviatoric components of the unperturbed stress are then given by definitions 5-2 as

$$
\begin{aligned}
& \mathrm{S}_{\mathrm{a}(1)}^{(1)}=\frac{1}{2} \frac{\mathrm{Pa}^{2} \lambda}{a_{0} h_{0}}-\frac{1}{6} \frac{F_{z} \lambda}{\pi a_{0} h_{0}} ; \\
& S_{a(2)}^{(2)}=\frac{F_{z} \lambda}{3 \pi a_{0} h_{0}} ; \\
& S_{a(3)}^{(3)}=-S_{a(1)}^{(1)}-S_{a(2)}^{(2)},
\end{aligned}
$$

and the unperturbed components of the strain rate are given by Eqs. 4-4 and 3$20 \mathrm{a}$ as

$$
\begin{aligned}
& \frac{\mathrm{d} \varepsilon \frac{(1)}{\mathrm{a}(1)}}{\mathrm{d} t}=\frac{1}{\mathrm{a}} \frac{\mathrm{da}}{\mathrm{dt}} \quad ; \\
& \frac{\mathrm{d} \varepsilon^{(2)}(2)}{\mathrm{d} t}=\frac{1}{\lambda} \frac{\mathrm{d} \lambda}{\mathrm{dt}} ; \\
& \frac{\mathrm{d} \varepsilon \frac{(3)}{\mathrm{a}(3)}}{\mathrm{dt}}=-\frac{\mathrm{d} \varepsilon \frac{(1)}{\mathrm{a}(1)}}{\mathrm{dt}}-\frac{\mathrm{d} \varepsilon^{(2)} \mathrm{a}(2)}{\mathrm{dt}} .
\end{aligned}
$$


Substituting Eqs. 5-12 and 5-13 into Eq. 5-7 yields the following two nonlinear differential equations:

$$
\begin{aligned}
& \frac{1}{\mathrm{a}} \frac{\mathrm{da}}{\mathrm{dt}}=\frac{3}{2} \mathrm{C}\left[\frac{1}{2} \frac{\mathrm{Pa}^{2} \lambda}{\mathrm{a}_{\mathrm{o}} \mathrm{h}_{\mathrm{o}}}-\frac{1}{6} \frac{\mathrm{F}_{\mathrm{z}} \lambda}{\pi \mathrm{a}_{\mathrm{o}} \mathrm{h}_{\mathrm{o}}}\right] \bar{\sigma}_{\mathrm{a}}^{\mathrm{n}-1,}, \\
& \frac{1}{\lambda} \frac{\mathrm{d} \lambda}{\mathrm{dt}}=\frac{3}{2} \mathrm{C}\left[\frac{\mathrm{F}_{\mathrm{z}} \lambda}{3 \pi \mathrm{a}_{\mathrm{o}} \mathrm{h}_{\mathrm{o}}}\right] \bar{\sigma}_{\mathrm{a}}^{\mathrm{n}-1},
\end{aligned}
$$

from which $a(t)$ and $\lambda(t)$ can be determined by numerical integration.

We now turn to the analysis of the stresses and strains in the perturbed geometry. The normal component of the stress perturbation $\sigma_{\varepsilon}^{(3)}$ (3) must be zero in order to be consistent with the thin-shell assumption that membrane stresses are much larger than normal stresses. Furthermore, the strain-rate perturbations are given in terms of $\varepsilon$ by Eqs. $4-4$ and $3-20 b$ :

$$
\begin{aligned}
& \frac{\mathrm{d} \varepsilon_{\varepsilon(1)}^{(1)}}{\mathrm{dt}}=\frac{\mathrm{d}}{\mathrm{d} t}\left(\frac{\varepsilon}{\mathrm{a}}\right) ; \\
& \frac{\mathrm{d} \varepsilon^{(2)}(2)}{\mathrm{dt}}=0 ; \\
& \frac{\mathrm{d} \varepsilon \frac{(3)}{\varepsilon(3)}}{\mathrm{dt}}=-\frac{\mathrm{d}}{\mathrm{dt}}\left(\frac{\varepsilon}{\mathrm{a}}\right) .
\end{aligned}
$$

Substitution of Eqs. 5-16 into Eq. 5-8 yields the single equation

$$
\frac{\mathrm{d}}{\mathrm{dt}}(\varepsilon / \mathrm{a})=\frac{3}{2} \mathrm{C} \mathrm{T}_{1 \mathrm{a}} \underset{\varepsilon(1)}{(1)}+\frac{3}{2} \mathrm{C}_{\varepsilon} \mathrm{T}_{2 \mathrm{a}}
$$


where

$$
\begin{aligned}
& \mathrm{T}_{1 \mathrm{a}}=\left[\begin{array}{ccc}
\frac{3}{2}(\mathrm{n}-1) \bar{\sigma}_{\mathrm{a}}^{\mathrm{n}-3} & \mathrm{~s} \\
& \mathrm{a}(1) & \mathrm{s}(1) \\
\mathrm{a}(1)
\end{array}+\frac{2}{3} \bar{\sigma}_{\mathrm{a}}^{\mathrm{n}-1}\right]- \\
& \left.\left[\frac{3}{2}(\mathrm{n}-1) \bar{\sigma}_{\mathrm{a}}^{\mathrm{n}-3} \mathrm{~s} \underset{\mathrm{a}(1)}{\mathrm{s}} \mathrm{a}_{\mathrm{a}(2)}^{(2)}-\frac{1}{3} \bar{\sigma}_{\mathrm{a}}^{\mathrm{n}-1}\right]\right]^{2} / \\
& {\left[\frac{3}{2}(\mathrm{n}-1) \bar{\sigma}_{\mathrm{a}}^{\mathrm{n}-3} \mathrm{~S}_{\mathrm{a}(2)}^{(2)} \mathrm{s}_{\mathrm{a}(2)}^{(2)}+\frac{2}{3} \bar{\sigma}_{\mathrm{a}}^{\mathrm{n}-1}\right]}
\end{aligned}
$$

and

$$
\begin{aligned}
\mathrm{T}_{2 \mathrm{a}}= & \bar{\sigma}_{\mathrm{a}}^{2 \mathrm{n}-2}\left[\frac{2}{3} \mathrm{~S}_{\mathrm{a}}^{(1)}(1)+\frac{1}{3} \mathrm{~s}_{\mathrm{a}}^{(2)}(2)\right] / \\
& {\left[\frac{3}{2}(\mathrm{n}-1) \bar{\sigma}_{\mathrm{a}}^{\mathrm{n}-3} \mathrm{~s}_{\mathrm{a}(2)}^{(2)} \mathrm{s}_{\mathrm{a}(2)}^{(2)}+\frac{2}{3} \bar{\sigma}_{\mathrm{a}}^{\mathrm{n}-1}\right] }
\end{aligned}
$$

The remaining equation necessary to complete the formulation comes from the equilibrium equation. Substituting Eq. 5-11 into Eq. 3-21 gives

$$
\underset{\sigma}{(1)}(1)=\frac{2 \mathrm{~Pa}^{2} \lambda}{\mathrm{a}_{\mathrm{o}} \mathrm{h}_{\mathrm{o}}} \varepsilon / \mathrm{a}+\frac{\mathrm{Pa}^{2} \lambda}{\mathrm{a}_{\mathrm{o}} \mathrm{h}_{\mathrm{o}}}+\frac{\partial^{2} \varepsilon / \mathrm{a}}{\partial \theta_{\mathrm{o}}{ }^{2}}+\left[\frac{\mathrm{Pa}^{2} \lambda}{2 \mathrm{a}_{\mathrm{o}} \mathrm{h}_{\mathrm{o}}}+\frac{\mathrm{F}_{\mathrm{z}}}{2 \pi \mathrm{a}_{\mathrm{o}} \mathrm{h}_{\mathrm{o}}}\right] \frac{\mathrm{a}^{2}}{\lambda^{2}} \frac{\partial^{2} \varepsilon / \mathrm{a}}{\partial \mathrm{z}_{\mathrm{o}}{ }^{2}} \cdot
$$

Substitution of Eq. 5-19 into Eq. 5-17 yields a single linear differential equation for the perturbation $\varepsilon\left(\theta_{0}, z_{0}, t\right)$. Once the zeroth-order approximation for $a(t)$ and $\lambda(t)$ are known from Eqs. 5-14 and 5-15, this equation can easily be integrated. 
VI. RESULTS AND DISCUSSION

\section{A. Materia1 Properties}

In order to illustrate certain features of the bulging analysis presented in the preceding sections, it is desirable to choose a representative set of material properties. In general, cladding conditions for which the constitutive equation 5-5 is applicable are those of high temperature and moderate strain rates. We have chosen here a set of data from D. F. Atkins' work [4] on the stress-rupture behavior of stainless steel tubes under internal pressure. The particular results we have used are for unirradiated Type 316 stainless steel with 10 to $15 \%$ cold work. Fitting the two material constants $\mathrm{C}$ and $\mathfrak{n}$ in Eq. 5-5 to Atkins' curves of stress versus strain rate leads to the constants given in Table $I$.

\section{Table I}

\section{Stainless Steel Properties Used in Equation 5-5}

\begin{tabular}{lcc} 
Temp, ${ }^{\circ} \mathrm{C}$ & $\mathrm{n}$ & \multicolumn{1}{c}{${ }^{\mathrm{C}}$} \\
538 & 16.37 & $1.36 \times 10^{-45}$ \\
649 & 8.59 & $6.70 \times 10^{-24}$ \\
760 & 5.37 & $1.94 \times 10^{-14}$
\end{tabular}

The data base for these constants covers temperatures from 538 to $760^{\circ} \mathrm{C}$ and strain rates from $10^{-6}$ to $10^{-3} \mathrm{hrs}^{-1}$. Although these strain rates are very low for application to accident analysis, comparison of Atkins' data with high-strain-rate Lensile test data of J. M. Steichen [5] shows that Atkins' results can probably be extrapolated up to $10^{-2} \mathrm{~s}^{-1}\left(36 \mathrm{~h}^{-1}\right)$ at the highest temperature $\left(760^{\circ} \mathrm{C}\right)$. Beyond this point, the mechanical behavior rapidly becomes independent of strain rate as work-hardening processes begin to dominate. of course for cladding temperatures above $760^{\circ} \mathrm{C}$, the strain-rate regime for which Eq. 5-5 is applicable is correspondingly greater.

In the present analysis, the onset of unstable bulge growth is coincident with the onset of unstable growth of either of the unperturbed parameters a or $\lambda$. If only the time to failure is of interest, and if the failure strains 
are large, Eqs. 5-14 and 5-15 can be integrated over the loading history to the point at which either a or $\lambda$ tends to arbitrarily large values. Due to the extremely rapid growth of strains near failure, the resulting time to failure is insensitive to the actual value of a or $\lambda$ chosen. For the case of constant-pressure loading of closed tubes at constant temperature, Eqs. 5-14 and 5-15 can easily be solved. Integrating these equations shows that the axial deformation $\lambda$ remains constant: equal to 1 , while the radius a(t) tends to infinity when time $t$ reaches

$$
\mathrm{t}=\frac{2^{\mathrm{n}}}{3^{\frac{\mathrm{n}+1}{2}}}\left[\frac{1}{\mathrm{nC}}\left(\frac{\mathrm{Pa}_{\mathrm{o}}}{\mathrm{h}_{\mathrm{o}}}\right)^{-\mathrm{n}}\right] \text {. }
$$

This equation, with the material constants from Table I, predicts times to rupture for Atkins' data (symbols) as shown by the curves in Fig. 3. Although the comparison is surprisingly good, it must be pointed out that the parameters in Table $\mathrm{I}$ are based on strain rates averaged over the life of each specimen.

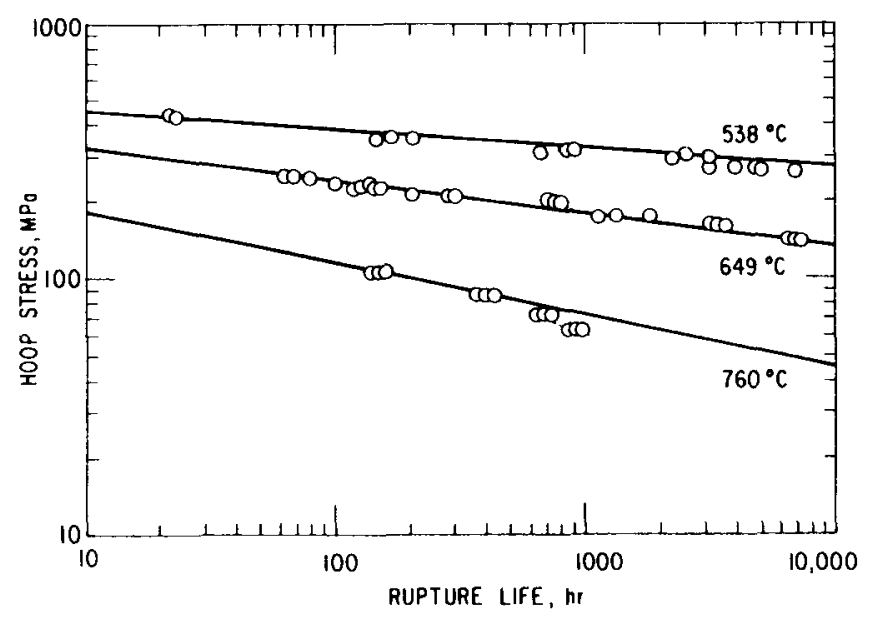

Fig. 3 Biaxial Stress-rupture Strength of 10-15\% Cold-worked Stainless Steel, Data (symbols) fron D. F. Atkins [4]. $(1 \mathrm{~h}=3600 \mathrm{~s})$

It is therefore impossible to distinguish between behavior governed by Eq. 5-5 and a more general constitutive equation of a form in which the right-hand side of Eq. 5-5 is multiplied by a material function $G(t)$. It is interesting to note however, that equations of this form are often employed to describe creep behavior when both primary creep and steady-state creep are important. 
It should also be noted that the deviation of the high-temperature data from the curve in Fig. 3 is real, as shown in other results from Atkins' report. This deviation probably represents a change in creep mechanisms at lower stresses.

\section{B. Bulge Behavior}

The behavior of the bulge displacement $\varepsilon$ is governed by Eqs. 5-17 and 519. For the moment we consider only the case in which the temperature is uniform so that $C_{\varepsilon}=0$. The spatial dependency of $\varepsilon$ can be eliminated from the equations by assuming a solution of the form

$$
\varepsilon / a=B(t) \cos \left(m \theta_{0}\right) \cos \left(\frac{\pi z_{0}}{L_{0}}\right) ; \quad m=0,1,2, \ldots \ldots,
$$

where $\mathrm{L}_{\mathrm{O}}$ is the length of the bulge as shown in Fig. 1B. Equation 6-2 can be viewed as either representing the growth of a single imperfection in the geometry with initial amplitude $\mathrm{B}(0)=\mathrm{B}_{\mathrm{O}}$ or as a Fourier component of a more general imperfection. Since the governing equations for $\varepsilon / a$ are linear, solutions of the form of $\mathrm{Eq}$. 6-2 can then be superimposed.

Substituting Eq. 6-2 and 5-11 into Eqs. 5-17 and 5-19 gives

$$
\frac{\mathrm{dB}}{\mathrm{dt}}=3 \mathrm{C} \mathrm{T}_{1 \mathrm{a}}(\mathrm{t}) \frac{\mathrm{Pa}^{2}}{\mathrm{a}_{0} \mathrm{~h}_{\mathrm{o}}} \lambda\left[1-\frac{1}{2} \mathrm{~m}^{2}-\frac{\mathrm{a}_{\mathrm{o}} \mathrm{o}_{\mathrm{o}}}{2 \mathrm{~Pa}^{2} \lambda} \sigma_{\mathrm{za}} \frac{\mathrm{a}^{2} \pi^{2}}{\lambda^{2} \mathrm{~L}_{\mathrm{o}}^{2}}\right] \mathrm{B} \text {. }
$$

Equation 6-3 determines the ratio of the amplitude of the bulge to the radius $a(t)$ of the uniform sections of the deformed cladding. Although this equation was derived for a particular constitutive equation, it should be noted that the term inside the square brackets will appear regardless of the constitutive equation since it comes from the equilibrium conditions Eq. 5-14. In the present analysis, the sign of the term governs whether $\mathrm{dB} / \mathrm{dt}$ in $\mathrm{Eq} \cdot 6-3$ is positive or negative, that is, whether or not the amplitude of an initial geometric imperfection will grow or shrink as the cladding deforms.

For pressure loading of a closed tube, $\sigma_{z a}$ is just $1 / 2$ the nominal hoop stress given by Eqs. 3-10 and 3-20a. In this case $\lambda=$ constant $=1$. Substituting these results into Eq. 6-3 shows that the only values of $\mathrm{m}$ that will lead to bulge growth are 0 and 1 . The case $m=0$ represents a bulge around the entire circumference, whereas the case $m=1$ represents a bulge around half of the circumference. If it is assumed that the smallest geometric 
imperfection that will grow provides the most likely nucleus for the bulge, Eq. 6-3 gives $\mathrm{m}=1$ and $\mathrm{L}_{\mathrm{o}} / \mathrm{a}=2.22$. This corresponds to a bulge on one side of the cladding with a length 1.11 times the deformed diameter. It is encouraging to note that this shape is consistent with the bulge formation that has been reported for burst tests of Type 316 Stainless Steel cladding [2].

We have written a small computer program to solve Eqs. 5-14, 5-15, and 6-3 numerically. Figures 4, 5, and 6 show results for dimensionless radial, axial, and bulge displacements, using the material properties for cladding at $760^{\circ} \mathrm{C}$ from Table I. The parameter $\lambda$ is defined by Eq. $2-2 c$, whereas $\hat{a}$ and $\hat{\varepsilon}$ are defined by

$$
\hat{a}=a(t) / a_{0} ; \quad \hat{\varepsilon}=B(t) / B_{0}
$$

A11 three figures use $a_{0}=2.73 \mathrm{~mm}, h_{0}=0.381 \mathrm{~mm}, P=19.26 \mathrm{MPa}$, and assume $\mathrm{m}=1$.

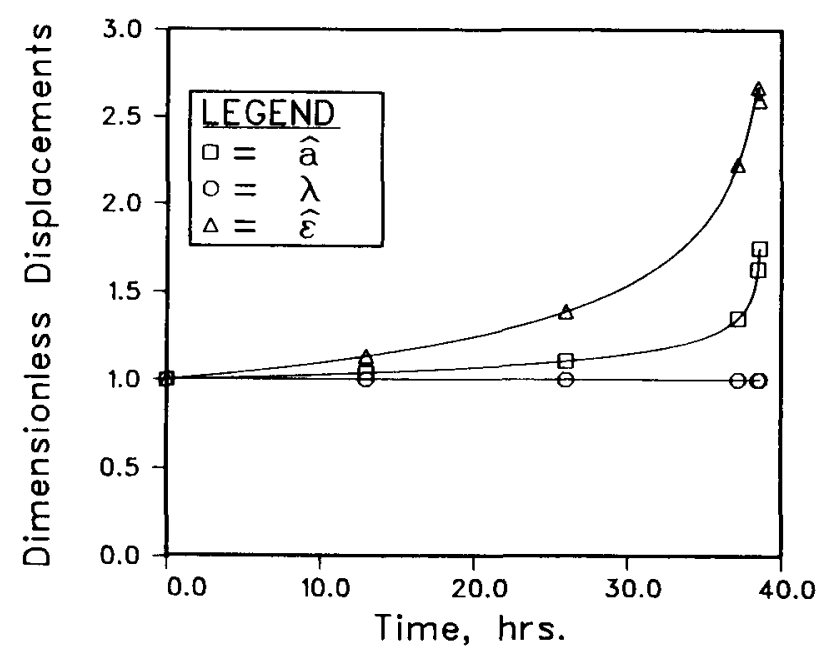

Fig. 4 Dimensionless Radial (a), Axial $(\hat{\lambda})$, and Bulge $(\hat{\varepsilon})$ Displacements of a Closed Tube for an Initial 0.0055-mm-amplitude Bulge of Length $10.0 \mathrm{~mm}$ and No Temperature Perturbation. (1 hr $=3600 \mathrm{~s})$ 


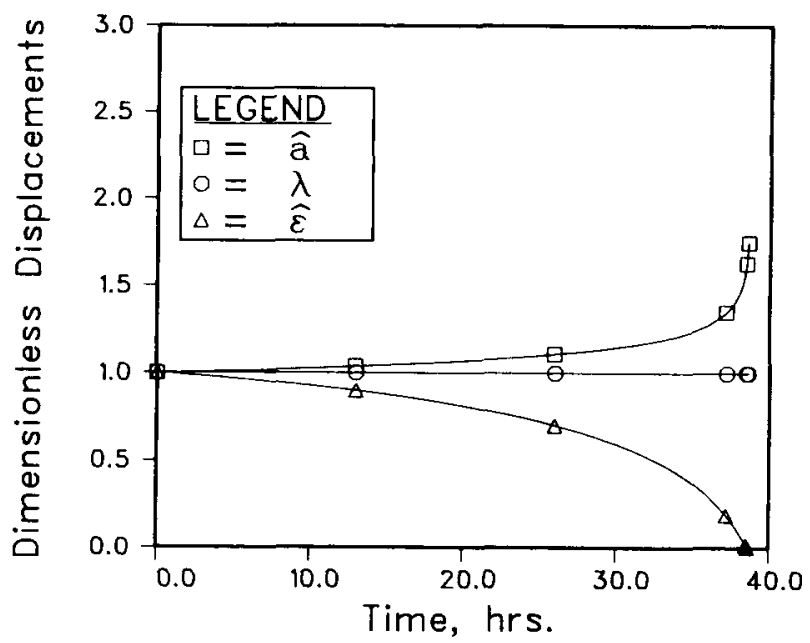

Fig. 5 Same as Fig. 4 Except with an Initial Bulge Length of $5.0 \mathrm{~mm}$. (1 hr= $3600 \mathrm{~s})$

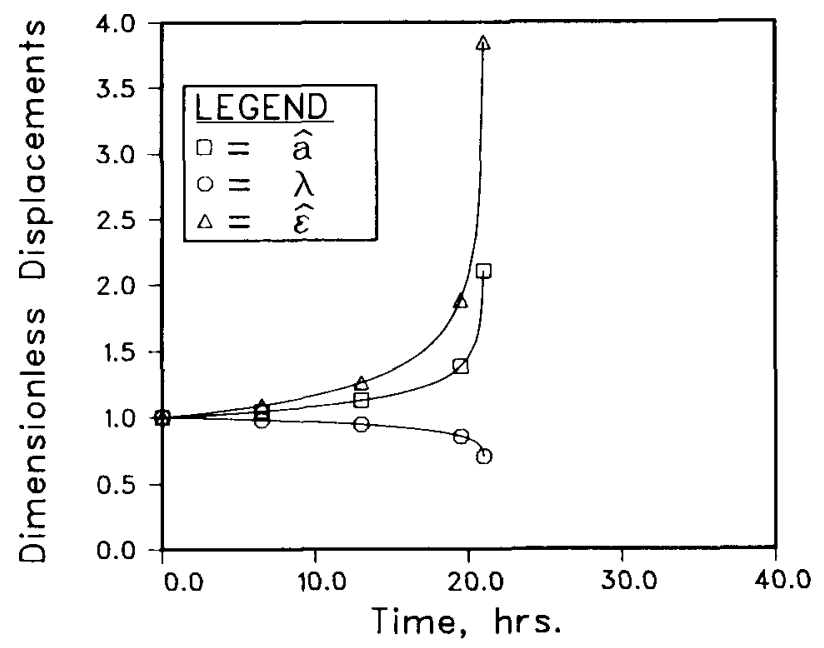

Fig. 6 Same as Fig. 4 Except for an Open Tube with No Axial Force. (1 hr $=3600 \mathrm{~s})$ 


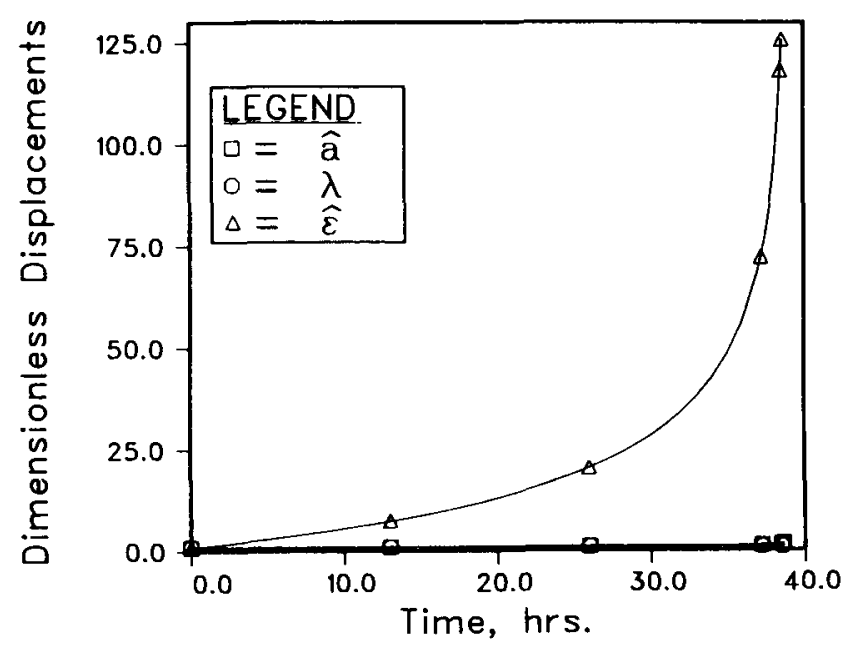
Fig. 7 Same as Fig. 4 Except with $10^{\circ} \mathrm{C}$ Temperature Perturbation. $(1 \mathrm{hr}=3600 \mathrm{~s})$

Figure 4 shows the behavior of a closed tube with $\mathrm{L}_{\mathrm{o}}=10.0 \mathrm{~mm}$. It is seen that $\lambda$ remains constant while the unperturbed radius grows unstably at $t=38.9 \mathrm{~h}\left(1.40 \times 10^{5} \mathrm{~s}\right)$. This is the same time as given by Eq. 6-1. The bulge displacement is also seen to grow. Figure 5 shows the behavior under similar conditions except that the initial imperfection is assumed to have a length $\mathrm{L}_{\mathrm{o}}=5.0 \mathrm{~mm}$. The relative amplitude of this imperfection is seen to shrink in this case as the tube deforms.

Figure 6 shows the deformation behavior when the axial stress $\sigma_{z a}$ is zero. The other conditions are the same as in Fig. 5. This type of loading, which may be more typical of cladding loading during overpower transients, considerably reduces the predicted failure time.

\section{Temperature Perturbations}

The effect of temperature perturbations on the behavior of local plastic instability enters the analysis through the perturbation $C_{\varepsilon}$ of the material constant $C$. The differences in the values of $C$ in Table $I$ suggest that very small temperature variations can lead to a significant value for $\mathrm{C}_{\varepsilon}$. In fact, it is usually assumed that $C$ can be represented by an exponential Arrheniustype equation:

$$
\mathrm{C}=\mathrm{C}_{\mathrm{o}} \exp \left(-\mathrm{T}^{*} / \mathrm{T}\right) \text {, }
$$


with an activation lemperature $\mathrm{T}^{*}$ approximately equal to the activation temperature for self-diffusion. Unfortunately, since the parameter $\mathrm{n}$ in Table I also varies somewhat with temperature, it is impossible to determine a meaningful value for $\mathrm{T}^{*}$ from the data. We choose instead to assume that $\mathrm{T}^{*}$ is equal to the activation temperature for self-diffusion, name1y, 33,700K. For small temperature variations $T_{\varepsilon}\left(\theta_{0}, a_{0}, t\right)$, about the mean temperature $T_{a}$, Eq. 6-5 shows that

$$
\mathrm{C}_{\varepsilon}=\mathrm{CT}^{*} \mathrm{~T}_{\varepsilon} / \mathrm{T}_{\mathrm{a}}^{2}
$$

Two sources of cladding-temperature perturbations that have been considered are those due to variations in the fuel-cladding gap and those due to local perturbations in the sodium cooling. Two-dimensional heat-transfer calculations have shown that the temperature perturbations due to variations in the fuel-cladding gap are expected to range from 1 to $5^{\circ} \mathrm{C}$ around the circumference. Although results are not presented here, it is noted that initial fuel eccentricity provides a source for bulge growth through $\mathrm{C}_{\varepsilon}$ in Eq. 5-17. As the bulge grows away from the fuel, however, the resulting decrease in local cladding temperature tends to stabilize the growth.

Larger Lemperature perturbations in the cladding are caused by local perturbations in the sodium cooling. Numerical calculations by Chuang, et al., [6] have shown that temperatures of cladding hot spots due to the hexagonal geometry vary from 1 to $5^{\circ} \mathrm{C}$, whereas those due to spacer wires are typically 10 to $20^{\circ} \mathrm{C}$. These temperature perturbations also act as a bulging source that behaves similar to initial geometric imperfections.

Our calculations show that temperature perturbations with 60-degree hexagonal periodicity cause initial bulge growth, but do not lead to unstable growth. This is because for $m=6$ the term in the square brackets in Eq. 6-3 is negative. On the other hand, temperature perturbations due to features such as wire spacers have Fourier components with $\mathrm{m}$ equal to 0 and 1 . Figure 7 shows an example of the bulge behavior for the same conditions as for Fig. 1 but with a temperature perturbation of $10^{\circ} \mathrm{C}\left[\left(\mathrm{T}_{E}=10 \cos \left(\theta_{0}\right) \cos \left(\pi \mathrm{z}_{0} / \mathrm{L}_{\mathrm{o}}\right)\right]\right.$. In both figures $B_{0}=0.002$. It is seen from the different scales in these figures that, for the parameters assumed, the effect of the temperature perturbation is much greater than that due to the initial geometric imperfection. 
VII. CONCLUSIONS

A perturbation theory of cladding failure by local plastic instability has been developed. The resulting equations that govern the growth of a geometric imperfection, or bulge, are linear and are easily solved once the zeroth-order solution for the deformation of a perfect cylinder is known.

For hydrostatic loading of a closed tube, the most likely local plastic instability corresponds to a bulge on one side of the cladding with a length of about 1.11 times the deformed diameter. If creep damage of the grain boundaries were sufficient to cause pin-hole failure, resulting in relief of loading early in the deformation, the bulge amplitude would remain small. If the loading were not relieved, failure would occur by plastic instability of the bulge and would be characterized by sudden rupture with a rip along the length of the bulge. In either case, the failure criterion should be based on the perturbed stresses and strains rather than the uniform stresses and strains. In the more general case of loading during overpower conditions, both hydrostatic and rigid mandrel like loading components may contribute. In this case, bulging can also take place if local plastic instability occurs due to the hydrostatic component of loading before uniform cladding expansion is sufficient to cause failure.

Changing the biaxiality of the loading from the 2:1 closed-tube state has been found to exert a considerable influence on the bulge growth dynamics. For instance, a stress state in which the axial stress is zero, which may be more typical of transient overpower conditions, significantly reduces the time to the onset of plastic instability.

Temperature perturbations have also been shown to affect bulge growth. The combination of large creep-activation energies and high temperature conditions typical of accident conditions make the growth dynamics sensitive to temperature perturbations as small as 5 to $10^{\circ} \mathrm{C}$. Sources for such temperature perturbations are variations in the fuel-cladding gap due to eccentric fuel pellets and local perturbations in the sodium cooling. Of these, the perturbations of coolant flow are the most important, both because the claddingtemperature variations due to eccentric fuel pellets are usually smaller than those due to flow perturbations and because bulge growth away from the fuel tends to decrease cladding temperatures, thus stabilizing the growth. 
Our calculations have shown that temperature perturbations in the cladding due to the $60^{\circ}$ (1.05 rad) hexagonal periodicity of the flow channels provide a source for bulging similar to the source provided by initial geometric imperfections, but do not lead to unstable growth. On the other hand, temperature perturbations due to features such as wire spacers, which have both axisymmetric components and components with $360^{\circ}(2 \pi \mathrm{rad})$ periodicity, do lead to unstable growth. Here, temperature perturbations with a magnitude of the order of $10^{\circ} \mathrm{C}$ are sufficient to dominate the effect of any anticipated initial geometric imperfections.

Numerical results have been presented in this report for cladding temperature and loading conditions under which creep strains are dominant. These strains have been modeled by a power-1aw constitutive equation. However, other calculations using the more general endochronic theory [7] of visco-elastoplasticity, show qualitatively similar results.

One of the other areas that is being investigated is an extension of the analysis to include a calculation of the rip area at failure. A knowledge of this area is important in determining the initial release of fission gas and/or molten fuel from the pin. It is currently thought that the opening up of the cladding at the failure site occurs with little additional deformation in the circumferential direction, since the loading there is rapidly relieved. Elastic recovery cannot be sufficient to explain the large areas that are observed in some cases. It therefore seems logical to seek neighboring shapes into which the bulge with a rip along its length can open with no additional stretching. If this is the case, it is not difficult to show that if the bulge amplitude at failure is zero, so is the rip area. As the bulge amplitude at failure increases, so does the rip area. Unfortunately, a detailed analysis of this behavior involves the solution of a set of hyperbolic partial differential equations. However, a reasonable estimate of the rip area might be made by assuming that the cladding opens up on both sides of a rip along the length of the bulge such that the edges of the rip do not change length. The maximum area can then be found by assuming that the rip edges form arcs of a circle, whose arc length is completely determined from the bulge amplitude prior to failure. 


\section{ACKNOWLEDGMENTS}

The authors wish to thank E. E. Gruber for helpful discussions during the development of this work and for his help with the computer-generated graphical presentation of numerical results. 
1. Thomas, G. R. and Field, J. H., "Oxide Fuel Behavior During Transient Overpower Conditions", American Nuclear Society Proceedings - Fast Reactor Fuel Element Technology, New Orleans, Louisiana, April 1315, 1971, pp. 679-697 (1971).

2. Hunter, C. W., Johnson, G. D., and Fish, R. L., Mechanical Properties During Simulated Overpower Transients of Fast Reactor Cladding Irradiated from 700-1000 $\mathrm{F}$, HEDL-TME-75-28 (June 1975).

3. Struik, D. J., Differential Geometry, Addison-Wesley Publishing Company, Inc., Reading, Massachusetts (1950).

4. Atkins, D. F., Stress-Rupture Behavior of Types 304 and 316 Stainless Steel Cladding in High-Temperature Static Sodium, AI-AEC-12976 (Sept. 1970).

5. Steichen, J. M., High Strain Rate Tensile Proeprties of $20 \%$ C. W. Type 316 Stainless Stee1, HEDL-TME-74-39 (June 1974).

6. Chuang, M. C., Kothmann, R. E., Pechersky, M. J., and Markley, R. A., Cladding Circumferential Hot Spot Factors for Fuel and Blanket Rods, Nuc1. Eng. Des., 35, 21-28 (1975).

7. Kramer, J. M. and Deitrich, L. W., Modeling of Cladding Plasticity Using The Endochronic Theory, Trans. Am. Nuc1. Soc., 22, 576 (1975). 
Distribution for ANL-77-95

Internal:

J. A. Kyger

A. Amorosi

D. W. Cisse1

B. R. T. Frost

R. G. Staker

C. E. Till

R. S. Zeno

R. Avery

J. F. Marchaterre

A. J. Goldman

H. K. Fauske

B. D. LaMar

D. Rose

I. Bornstein

R. H. Sevy

P. A. Lottes

L. Baker

B. A. Korelc (4)

L. W. Deitrich (10)

J. M. Kramer (10)

E. E. Gruber

C. C. Meek

T. H. Hughes
R. J. DiMelfi

S. H. Fistedis

D. R. Ferguson

C. E. Dickerman

R. A. Nol and

W. C. Lipinski

T. C. Chawla

H. J. Petroski

R. F. Kulak

H. L. Schreyer

J. M. Kennedy

D. H. Cho

F. E. Dunn

W. L. Wang

H. U. Wider

A. B. Rothman

A. E. Klickman

A. E. Wright

H. H. Hummel

P. B. Abramson

R. E. Henry

D. H. Lennox

J. H. Tessier

D. R. Pedersen
J. C. Herceg

T. Ariman

M. A. Grolmes

D. Stah 1

R. B. Poeppe 1

M. C. Billone

D. R. Diercks

L. A. Neimark

F. L. Yaggee

H. Yamada

A. Purohit

H. M. Chung

V. Z. Jankus

J. T. Madel 1

R. A. Valentin

C. I. Yang

C. H. Yin

C. M. Walter

L. C. Walters

A. B. Krisciunas

ANL Contract File

ANL Libraries (5)

TIS Files (6)

\section{External:}

DOE-TIC, for distribution per UC-79p (282)

Manager, Chicago Operations Office

Chief, Chicago Patent Group

Director, DOE-RRT (2)

Director, Reactor Programs Div., DOE-CH

Director, CH-INEL

President, Argonne Universities Association

Reactor Analysis and Safety Division Review Committee:

W. Kerr, U. Michigan

M. Levenson, Electric Power Research Inst.

S. Levy, S. Levy, Inc.

R. B. Nicholson, Exxon Nuclear Co., Inc.

D. Okrent, U. California, Los Angeles

N. C. Rasmussen, Massachusetts Inst. Technology

S. Baron, Burns and Roe, Inc. 